Supporting Information for:

\title{
Catalytic Synthesis and Structural Charaterizations of Highly Crystalline Polyphenylacetylene Nanobelt Array
}

Wei Liu, ${ }^{*}$ Zhi-Min Cui,${ }^{*}$ Qiang Liu, ${ }^{*}$ Dong-Wei Yan, Jing-Yi Wu, Hui-Juan Yan, Yun-Long Guo, Chun-Ru Wang, * Wei-Guo Song, * Yun-Qi Liu, Li-Jun Wan

Beijing National Laboratory for Molecular Sciences, Chinese Academy of Sciences, Beijing 100080,

${ }^{\ddagger}$ Graduate School of Chinese Academy of Sciences, Beijing 100039, P. R. China,

crwang@iccas.ac.cn; wsong@iccas.ac.cn.

\section{Contents}

S1. The preparation of copper catalysts

S2. The SEM study of the PPA dense layer

S3. AFM study of individual PPA nanobelt

S4. I-V characterization of the PPA nanobelts. 


\section{S1. The preparation of copper catalysts}

The copper nanoparticles were delicately prepared to avoid exposing to oxygen in whole producing process. First, $200 \mathrm{ml}$ distilled water was heated to $100^{\circ} \mathrm{C}$ and boiled for ten minutes to remove the dissolved oxygen in water. After the water temperature was decreased to $60{ }^{\circ} \mathrm{C}$, then $0.5 \mathrm{~g} \mathrm{CuNO}_{3}$ and $0.10 \mathrm{M}$ ascorbic acid was added to the water one after the other. The mixed solution was magnetically stirred and then a drop of $\mathrm{NaBH}_{4}\left(5.0 \times 10 \mathrm{M}^{-4}\right)$ was added inside the solution. Immediately, the solution was changed to red color along with the copper nanoparticles being produced.

After the production of copper particles, they were gathered by centrifugation to the bottom of the centrifugal tube. Most extra water on the upper part of the centrifugal tube was carefully taken away, but enough water was still maintained on the tube to protect the copper nanoparticles from exposure to air. Afterwards, the catalysts were ultrasonic treated to re-disperse in water and then transferred into the autoclave together with water. High pure Ar (99.999\%) was used to purge the autoclave for removing the water and drying the catalysts. SEM study of the copper nanoparticlese revealed regular crystalline structure of these copper particles (Figure S1a), and EDS spectrometry of the sample shows only little trace of oxygen existing due to the copper oxides.

In a control experiment, the copper nanocrystal was deliberately exposed to air for about 1 day. It was revealed the catalysts changed color from red to black, and lose its activity for catalyzing PPA polymerization. 

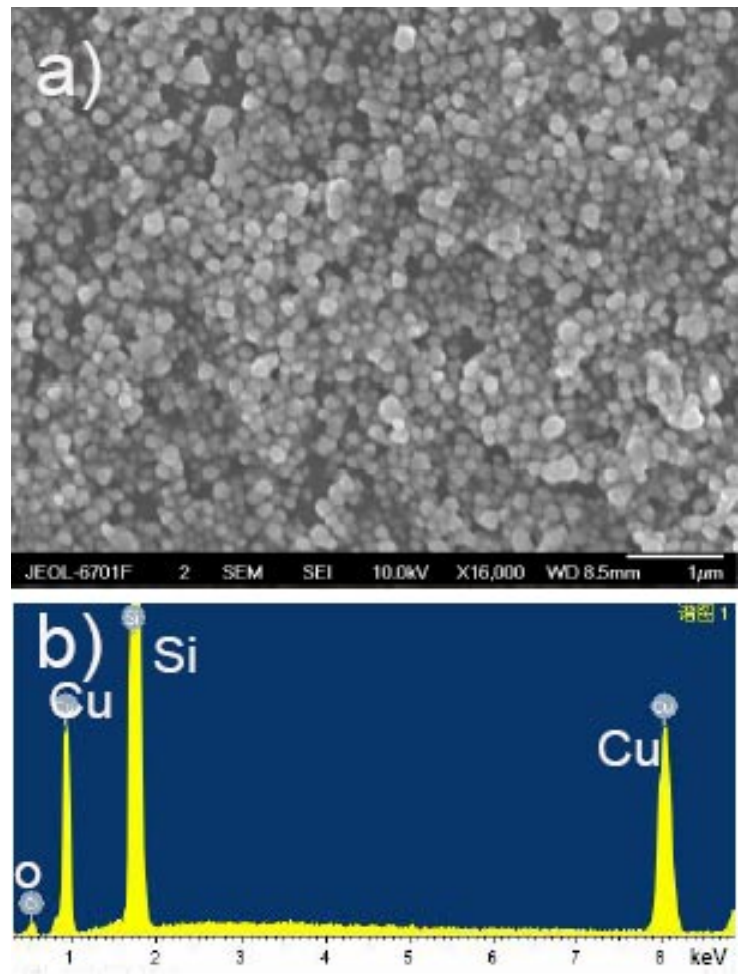

Figure .S1 a) SEM image of copper nanoparticles. b) EDS spectrum of the copper particles

\section{S2. The SEM study of the PPA dense layer}

The dense layer of PPA shown in Fig. 1a-b was studied by high-resolution SEM. As shown in Fig. S2, the high magnification SEM image indicates that the dense layer was composed of some tightly bundled PPA nanobelts that wrap the copper particles tightly.

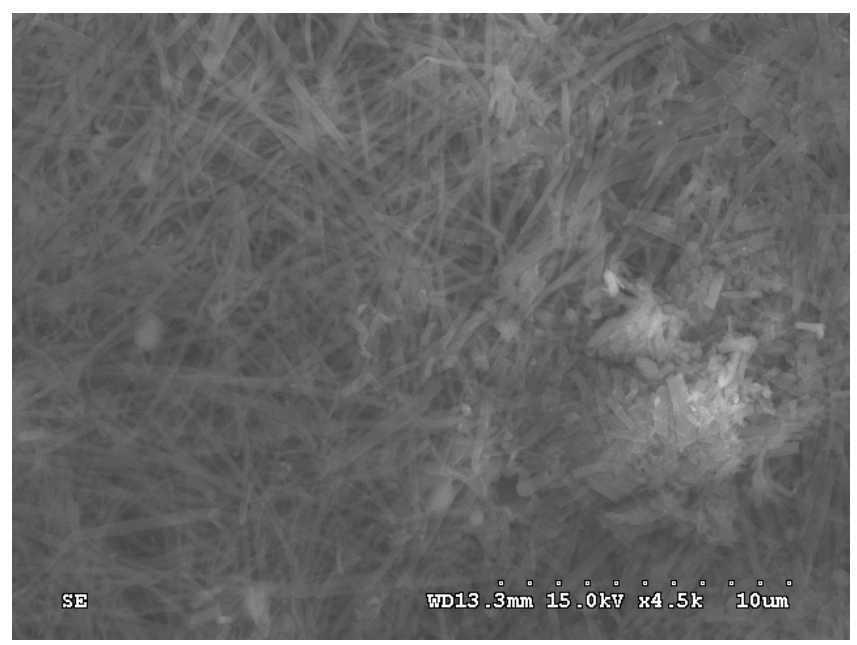

Figure S2. Magnification SEM image of the dense layer of PPA. 


\section{S3. AFM study of individual PPA nanobelt}

The sample was dispersed on silicon substrate, and AFM was performed to study the dimension of a single PPA nanobelt with a Nano IIIa (Veeco company, USA) by using a hard silicon cantilever tips (spring constant, $\sim 40 \mathrm{~N} / \mathrm{m}$ ) in a tapping mode. The morphological AFM image shown in Fig. S3 (a) clearly demonstrated the belt-like conformation of a single PPA nanobelt. According to the section analyzing results along the line $\mathrm{MN}$, the width of the single PPA nanobelt was measured to be about $135 \pm 5 \mathrm{~nm}$, and the height is about $24 \pm 3 \mathrm{~nm}$.
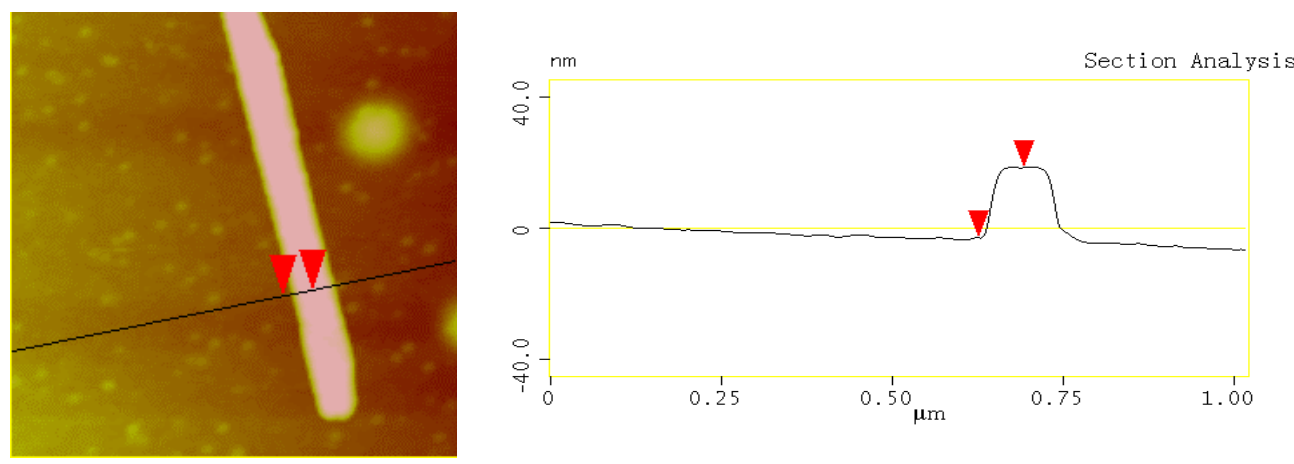

Figure S3. AFM image of a single PPA nanobelt. The width was about $135 \pm 5 \mathrm{~nm}$, and the height is about $24 \pm 3 \mathrm{~nm}$.

\section{S4. I-V characterization of the PPA nanobelts.}

A piece of the PPA array $\left(1 \mathrm{~mm}^{2}\right)$ was put into $20 \mathrm{ml}$ ethanol. After ultrasonic treatment for about 20 minutes, we drop a dip of the liquid on a $\mathrm{SiO}_{2}(500 \mathrm{~nm} \mathrm{SiO} 2$ covering on a $1 \mathrm{~cm} \times 1 \mathrm{~cm} \mathrm{Si}$ template) substrate on which an $8 \times 8$ array of $1 \mu \mathrm{m}$-bridged gold electrodes exist by thermally evaporating technique. After PPA dispersing on electrodes, we used a microscope to choose a suitable electrode pair in which a single PPA nanobelt bridges 2-electrodes and forms a single belt device. Then we measured the I-V curve of this device using a probe station (Wentworth, MP1008) and a semiconductor parameter analyzer (HP 4140B).

For iodine doping samples, the PPA nanobelts were lightly doped by exposed them to the iodine vapor with different doping times before they were dispersed on electrodes. 